\title{
Éxito del aprendizaje complejo a través del pensamiento estratégico
}

Pedro Fuentes-Duráa y Elena De la Poza Plaza ${ }^{b}$

aEscuela Técnica Superior de Ingeniería del Diseño, pfuentes@iqn.upv.es y ${ }^{\text {b}}$ Facultad de Administración y Dirección de Empresas, elpopla@esp.upv.es.

\begin{abstract}
This work deals with the development of students' strategic thinking, understood as a specific competence that fosters the complex learning in the university context.

Firstly by analyzing the theoretical framework of strategic thinking we identify the tools that promote its development.

Secondly, we show how the implementation of strategic thinking is useful and necessary in the pursuit of complex learning. The development of the complex learning is assessed through the strategic thinking in a semester course, with over 20 years' experience taught at the School of Design Engineering of the UPV since 2005, the EPS (European Project Semester). The program is characterized by multidisciplinary faculty and students, plus the internationality of students working on team projects. In this context of complexity, learning and attainment of skills and abilities of individuals, it is provided through a set of activities and techniques that develop the strategic thinking of the individual in the organization to which it belongs.
\end{abstract}

Keywords: strategic thinking, complex learning, University, European Project Semester, multidisciplinary.

\section{Resumen}

En este trabajo se describe el proceso de desarrollo del pensamiento estratégico del alumno, entendido como una competencia específica facilitadora del aprendizaje complejo en el contexto universitario.

En primer lugar se analiza a través de la literatura el pensamiento estratégico, las herramientas a través de las cuales se potencia y adquiere éste. 
Éxito del aprendizaje complejo a través del pensamiento estratégico

En segundo lugar, se muestra como la implementación del pensamiento estratégico es útil y necesaria en la consecución del aprendizaje complejo. Para ello se valora la generación del aprendizaje complejo a través del pensamiento estratégico en un curso semestral, con más 20 años de experiencia impartido en la Escuela Técnica Superior de Ingeniería del Diseño de la UPV desde 2005, el EPS, (European Project Semester) caracterizado por la multidisciplinariedad del profesorado y del alumnado, unido a la internacionalidad de los alumnos que desarrollan proyectos en equipo. En este contexto de complejidad, el aprendizaje como adquisición de competencias y habilidades de los individuos, se facilita a través de un conjunto de actividades y técnicas que desarrollan el pensamiento estratégico del individuo en la organización a la que pertenece.

Palabras clave: pensamiento estratégico, aprendizaje complejo, Universidad, European Project Semester, multidisciplinariedad

\section{Introducción}

\subsection{El contexto}

La Universitat Politècnica de València (UPV) está trabajando en la revisión de la definición de las competencias (fundamentalmente las competencias transversales) de las titulaciones oficiales que se imparten actualmente para tratar de medir y garantizar la adquisición de las dichas competencias. Las titulaciones impartidas en la UPV se diseñaron en torno a un catálogo de competencias, cuyo alcance es la meta formativa de cada titulación. Para validar este grado de alcance se dispone de experiencia en el caso de las competencias específicas; sin embargo, en el caso de las competencias transversales la experiencia es mucho menor. A esta dificultad se suma el hecho de que las competencias transversales suelen validarse a través de varias asignaturas, apareciendo un nuevo reto evaluativo. La UPV realizó, durante el verano de 2014, una serie de reuniones informativas con el fin de preparar las acciones a realizar y actualmente existe un grupo de trabajo para cada una de las competencias transversales listadas en su proyecto institucional. La UPV considera tres vías principales para la adquisición de competencias: los planes de estudios, los trabajos fin de grado (o máster) y las actividades extracadémicas.

Por otra parte, la Escuela Técnica Superior de Ingeniería del Diseño (ETSID) de la UPV lleva a cabo un proceso de optimización continua de las titulaciones impartidas en la misma, especialmente en lo referente a metodologías docentes que mejoren el aprendizaje activo y las oportunidades de aprendizaje. En los últimos años, ETSID recibe más de 250 estudiantes de intercambio cada curso lo que exige una oferta académica con valor añadido 
para los estudiantes extranjeros. Además, en los últimos 10 años, casi dos mil alumnos de la ETSID han disfrutado de un periodo en otra Universidad. Pese a ser una cifra espectacular, sobre el volumen global de alumnos de la ETSID no lo es, lo que significa que muchos egresados no han realizado estancias en otros centros. Por ello, la ETSID considera la internacionalización en casa un elemento inexcusable en su actividad. El European Project Semester (EPS) pretende contribuir de manera positiva a las intenciones de la UPV en general y de la ETSID en particular. Directamente, mediante el impacto en un número importante de estudiantes e, indirectamente, al hacer más atractiva la oferta para los estudiantes extranjeros y al contribuir al desarrollo profesional de los profesores.

\subsection{Pensamiento estratégico}

El pensamiento estratégico (PE) se define como la capacidad del individuo para reconocer las oportunidades y amenazas del entorno, y capacidad para fijar un objetivo que a través de los recursos disponibles sea alcanzado, generando una mejora personal, o bien de la organización en la que se integra (Bonn, 2001). Tradicionalmente se ha asociado el desarrollo del PE a la enseñanza de titulaciones relacionadas con la empresa, o bien a la adquisición de dicha competencia a través de la práctica profesional y aplicación de competencias técnicas del puesto de trabajo. Sin embargo, es posible desarrollar el PE y es necesario para fomentar la creatividad, e innovación en las formas de trabajar, en los modelos de negocio, productos y servicios de cualquier mercado (Christensen, 2007). El PE refleja la capacidad que el individuo tiene de investigar, interpretar y planificar a partir de la información disponible generando futuros escenarios, fruto de los cambios tanto en la organización a la que pertenece como los cambios en los agentes externos al individuo y a la propia organización. Estas capacidades que conforman el PE se pueden desarrollar, de ahí la necesidad de su estudio e implementación en la educación universitaria (Goldman, 2009).

Se puede considerar el PE como un tipo diferenciado de pensamiento, pero relacionado con el pensamiento no lineal porque ambos son futuristas y tolerantes al error. Grant (2013), discutió las diferentes fases en la evolución de la gestión estratégica desde la planificación financiera anual básica de los años 50 donde el éxito dependía del conocimiento de los productos, mercados y competidores al PE del siglo XXI. Hamel (2005) señaló que las estrategias suelen aparecer en el ritual de planificación anual. El punto de partida para la estrategia futura es la estrategia actual, con mejoras incrementales. Las oportunidades están en todas partes, pero las empresas suelen quedarse en segmentos y territorios conocidos. No todas las planificaciones son estratégicas, aunque se denominen así. En realidad muchos planes estratégicos son tácticos u operacionales y tienden a ser continuaciones del status quo. Sharifi (2012) arguyó que el PE puede ser más práctico en situaciones complejas y

\section{(cc) EY-NC-ND 2015, Universitat Politècnica de València}


competitivas porque tiene implicaciones significativas para el diseño de procesos de planificación. La planificación estratégica sucede tras el PE. El PE no es meramente pensar acerca de la estrategia sino el uso de analogías y similitudes para desarrollar nuevas ideas creativas y diseñar acciones basándose en nuevos aprendizajes, creando una perspectiva integrada.

Probablemente, el modelo más influyente de PE es el formulado por Liedtka (1998). Esquemáticamente, propone cinco elementos:

A. Perspectiva sistémica: Un pensador estratégico tiene un modelo mental del sistema completo de creación de valor de principio a fin, y entiende las interdependencias dentro de la cadena (impacto, relaciones, niveles, perspectivas, alianzas).

B. Intención estratégica: El PE permite a los individuos centrar su atención y concentrarse tanto como sea necesario para conseguir su objetivo.

C. Inteligencia oportunista: El PE invoca la capacidad de ser inteligentemente oportunista, o abierto a nuevas experiencias, lo que permite a las personas sacar partido de estrategias alternativas que pueden emerger en un medio ambiente rápidamente cambiante.

D. Pensar en el tiempo: El PE conecta el pasado con el presente y enlaza con la creación del futuro y la renovación.

E. Impulsado por las hipótesis: Las actividades centrales en el PE son la generación y comprobación de hipótesis. Es creativo y crítico a la vez, de una manera compleja.

Es importante distinguir entre el desarrollo del pensamiento estratégico que el individuo lleva a cabo a través de actividades tales como seminarios, entrevistas, visitas, planes de futuro, análisis de expectativas... y el desarrollo del pensamiento estratégico en el equipo, como proceso de cambio que persigue un fin, y cuya acción debe ser constantemente controlada, redirigida, y alimentada por todos los miembros del grupo.

El presente trabajo se centra en la formulación de estrategias, y en consecuencia actividades, que refuerzan el desarrollo del pensamiento estratégico grupal, y por tanto lleven al grupo no solo a la consecución de sus objetivos, en términos de resultados académicos o profesionales, sino al desarrollo de aptitudes y habilidades.

\subsection{European Project Semester}

EPS es un paquete formativo con formato de proyecto basado en el aprendizaje (Project Based Learning, PBL), con participantes de diferentes titulaciones trabajando en equipos multinivel, multidisciplinares y multiculturales (http://www.europeanprojectsemester.eu). 
PE requiere investigación, análisis y prospectiva, por lo que su aprendizaje no se puede restringir a clases teóricas. En este sentido, son ya muchas las universidades que incluyen situaciones simuladas, en periodos cortos de tiempo con grupos estándar de estudiantes.

En EPS los estudiantes son expuestos ante actividades características de creación y desarrollo de equipos, mejora de la comunicación, innovación, marketing y emprendimiento. Para ello, se articula un portafolio de atractivos proyectos multidisciplinares con una guía de buenas prácticas y sesiones con diferentes actores de interés (personal de universidad, empresas locales, instituciones, consultores expertos, estudiantes, etc.). A su vez, se emplean una serie de canales para la comunicación, en todo momento buscando la máxima eficiencia.

EPS es una alianza estratégica innovadora, coordinada y sostenible de 17 instituciones de educación superior de 12 países diferentes con objetivos comunes pero una alta diversidad. Se encuentra en continuo crecimiento cuyos socios imparten EPS aportando sus singularidades y participando en el intercambio de estudiantes, profesores y experiencias. EPS se encuentra en la línea de Education and Training 2020 y 2011 EU modernisation Agenda.

EPS promueve el desarrollo profesional del personal universitario, que rediseña regularmente la configuración de los cursos y los proyectos, al tiempo que establece un interesante puentes entre las diferentes partes interesadas en la educación superior.

Asimismo, EPS es un programa académico complejo diseñado para la adquisión de competencias y habilidades por parte de los estudiantes. Proporciona un conocimiento multidisciplinar lo que consideramos muy valioso para su empleabilidad. Participan instituciones públicas y agentes industriales en la realización de proyectos altamente aplicables desde el punto de vista científico y/o tecnológico, lo que mejora el acceso de los estudiantes a un mercado laboral cada vez más competitivo y exigente con las competencias.

EPS promueve el autoconocimiento de los estudiantes. Si conoces mejor tu personalidad y tus competencias (motivaciones, deseos, formas de trabajar, puntos fuertes, debilidades) serás más capaz de entender a los demás. Esto contribuye a la cultura de empatía y tolerancia y permite el desarrollo personal y profesional.

Los estudiantes implicados han de desarrollar y documentar un proyecto que será evaluado por los participantes, así como profesores y externos (agentes económicos/industriales) para incrementar su prestigio, competitividad y valor académico.

EPS presta mucha atención al desarrollo de competencias interpersonales y comunicativas. Se desarrolla completamente en inglés (junto a la opción de estudiar la lengua y cultura

\section{(cc) EY-NC-ND 2015, Universitat Politècnica de València}


local) proporcionando un valor añadido a los estudiantes al facilitar y alentar el entendimiento intercultural.

EPS incide en el carácter emprendedor y otras competencias clave del alumnado, enfatizando en las que se consideran relevantes para la empleabilidad y la cohesión de la sociedad, particularmente al aumentar las oportunidades de movilidad del alumnado y al fortalecer la cooperación entre el mundo educativo y el entorno socioeconómico.

EPS es innovador en su contenido, pero aún más en su formato porque proporciona una metodología multiactor que implica un innovador proceso educativo que incluye el empleo de las nuevas tecnologías. EPS también innova en el establecimiento de nuevas alianzas y redes, haciendo hincapié en la comunicación y el intercambio de experiencias. Resumiendo, proporciona "new skills for new Jobs".

\section{Objetivos}

El principal objetivo de este trabajo es identificar y analizar la necesidad e importancia del desarrollo del pensamiento estratégico del alumno, cualquiera que sea su disciplina a través de actividades y metodologías activas desarrolladas en equipo que conducen al aprendizaje del individuo, y al desarrollo de sus habilidades y capacidades. El EPS ha sido el principal banco de pruebas durante los últimos 10 años. Independientemente del modelo pedagógico escogido, la motivación de los estudiantes necesita tareas interesantes y retadoras que le permitan, con un esfuerzo razonable, sentirse satisfecho de lo realizado. EPS proporciona un enfoque PBL caracterizado por la diversidad (multidisciplinariedad, internacionalidad, etc.) de alumnos y profesores trabajando en retos complejos en el contexto universitario.

Un segundo objetivo asociado es evaluar el impacto del EPS en las competencias transversales: Aplicación y pensamiento práctico, análisis y resolución de problemas, Innovación, creatividad y emprendimiento y comunicación efectiva que soportan el pensamiento estratégico.

El tercer objetivo es la contribución al desarrollo profesional de los profesores de la ETSID en el campo de la innovación educativa, con especial referencia al pensamiento estratégico.

\section{Desarrollo de la innovación}

\subsection{Pensamiento estratégico}

Existen 6 razones fundamentales para prestar atención al pensamiento estratégico: 
A. Los procesos de innovación requieren pensamiento estratégico tanto en la fase de generación de ideas, como en la evaluación de oportunidades y selección de proyectos y, por supuesto, en el desarrollo de los mismos (AENOR, 2006).

B. Un aprendizaje complejo sólo se alcanza a través de experiencias de diseño que incluyan un conocimiento del pasado, una atención al presente y una proyección hacia el futuro (Llovet, 1979).

C. Los estudiantes están participando de un cambio en el paradigma de la educación superior donde el aprendizaje se basa cada vez más en proyectos/problemas, empresas, internet y competencias (Vest, 2012).

D. La generalización de los procesos de gestión estratégica unida a la necesidad de adaptación al cambio hace que tanto en la dimensión personal como profesional sea cada vez más temprana la edad en que se hacen necesarias habilidades estratégicas (Oltra et al., 2014).

E. La empleabilidad ha adquirido un papel crucial en el ámbito universitario, y los empleadores buscan personas que sean capaces de interpretar sus estrategias y contribuir a su construcción y desarrollo (Sweet y Meiksins, 2013).

F. Por otro lado, el auge del emprendimiento insta a la formación de los estudiantes en la estrategia empresarial, al tiempo que se cultiva una actitud emprendedora y un espíritu emprendedor mediante la creación de escenarios propicios y exposiciones que les aproximen a esta cultura (Edwards-Schachter et al., 2015).

\subsection{Aprendizaje complejo}

El aprendizaje complejo (que proporciona competencia experta) necesita del éxito de los siguientes componentes (Castejón, 2006): la habilidad intelectual, la organización del conocimiento, la motivación, el uso de estrategias de aprendizaje, y la percepción del contexto en que se lleva a cabo este proceso. El tipo de inteligencia que parece más relacionado con la adquisición de conocimientos es la inteligencia práctica. La calidad del conocimiento (lo coherente, organizado, integrado y diferenciado a la vez) es el elemento que mayor influencia tiene sobre la adquisición del conocimiento y las habilidades. El uso deliberado de estrategias durante el estudio tiene una influencia directa sobre la adquisición del conocimiento. El modo en que definamos el concepto de estrategias parece determinar el grado en que éstas se relacionan con los resultados del aprendizaje. El uso real y deliberado de estrategias influye más en la adquisición del conocimiento que el tiempo total dedicado al estudio. El tipo de actividad realizada de forma deliberada, consciente y con esfuerzo, tiene efectos positivos sobre el desarrollo de la competencia experta (Ward, 2013) porque influye directamente en la motivación, que es la condición más importante para la práctica. El aspecto motivacional que está sistemáticamente relacionado con el aprendizaje

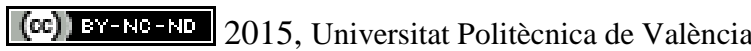


Éxito del aprendizaje complejo a través del pensamiento estratégico

y la adquisición de conocimientos es cercano a la motivación de logro (esfuerzo continuado por conseguir una meta y con el objetivo de mejorar la ejecución).

Un ambiente rico y variado de aprendizaje, en el que se combinan explicaciones, discusiones en grupo, aprendizaje cooperativo y trabajo independiente, está relacionada positivamente con la adquisición del conocimiento y las habilidades. La implicación instruccional para el desarrollo de la competencia experta parece clara: hay que proporcionar ambientes ricos en aprendizaje, que estimulen.

La implementación del pensamiento estratégico es útil y necesaria en la consecución del aprendizaje complejo. El EPS, caracterizado por la multidisciplinariedad del profesorado y del alumnado, unido a la internacionalidad de los alumnos que desarrollan proyectos en equipos proporciona un contexto de complejidad donde el aprendizaje como proceso de adquisición de competencias y habilidades de los individuos que integran cada equipo, se hace posible a través de un conjunto de actividades y técnicas que describiremos a continuación.

\subsection{Herramientas para el PE individual}

Linkow (1999) considera que las competencias del PE se pueden aprender a través de lluvia de ideas y planificación de escenarios para visualizar posibles futuros. Sugiere la realización de experimentos de bajo riesgo a pequeña escala para generar un rango de observaciones que han demostrado ser útiles en muchos campos.

La manera más simple para trabajar el PE con los estudiantes es considerar la siguiente simplificación del patrón de pensamiento de Drucker en tres etapas (Zand, 2010): 1) Hacer preguntas penetrantes, 2) Cambiar el encuadre y simplificar, 3) Considerar asunciones alternativas. Evidentemente, las preguntas han de ser respondidas por quien conozca la realidad actual y futura y por lo tanto pueden generar y evaluar un conjunto creativo de opciones estratégicas.

Para el desarrollo del PE individual:

A. Creamos un proceso de inmersión en el tema (clases, investigación, sesiones generativas) y en el contexto (seminarios, visitas, entrevistas, observaciones) al tiempo que se desarrollan actividades de construcción del equipo. Esto hace posible una comprensión holística.

B. Cultivamos la creatividad individual (colores, objetos, relajación, asociación libre, analogías, mapas mentales).

C. Generamos una visión hacia el futuro (futuro perfecto, preparación para el cambio, pensamiento en tres horizontes).

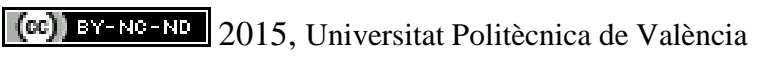

Congreso IN-RED (2015) 
El desarrollo del PE requiere el examen de individual de las creencias y asunciones de como funciona el mundo. Uno de los principales impedimentos para adoptar un pensamiento abierto y un estilo crítico/preguntón imprescindibles para el PE es la tendencia a rechazar lo que no es tangible o conocido. Identificar y comprender nuestro modelo mental comienza con una introspección que haga aflorar nuestra imagen de la realidad y permita criticarla. Esto conduce a un pensamiento abierto que aumenta la sensibilidad y el interés por entender a los demás, lo que implica conceptos esenciales para un exitoso PE: habilidades de escucha, resonancia, inteligencia emocional.

En coherencia con lo expuesto, se estimula a los estudiante para acelerar el proceso creativo, especialmente cuando se identifican bloqueos, siguiendo las sugerencias de Sloan (2006): En lugar de decir no, formula una pregunta; Formula preguntas para profundizar; Registra pensamientos, sentimientos y nuevas preguntas en un blog (diario o cualquier otra vía); Tómate tiempo tomar nota de los sentimientos que acompañan a tus pensamientos; Reflexiona acerca de las cosas que provocan preguntas o sentimientos fuertes; Dibuja (o diseña) tus ideas estratégicas en lugar de escribirlas; Diversifica y mejora tu verbal engagement; Escribe palabras y analogías que ilustren tus asunciones y creencias sobre los problemas estratégicos.

\subsection{Herramientas para el PE en equipo}

Intentamos desarrollar el PE individual y el PE en equipo. De este modo los estudiantes entienden la influencia del contexto organizacional en la conducta y el pensamiento individual; y la contribución de los individuos al equipo. La evaluación es una parte del ciclo de entrada, transformación y resultado, en la que la percepción, la comprensión y el razonamiento alcanzan el máximo nivel (Wells, 2001). El PE articula un ciclo con preguntas como ¿Qué parece que va a pasar? ¿Qué posibilidades tenemos? ¿Qué vamos a hacer al respecto? Todas ellas soportan el diálogo para elegir la dirección estratégica.

Para el desarrollo del PE grupal creamos estructuras, procesos y sistemas que:

A. Hagan crecer el dialogo estratégico dentro del equipo (Gordon/Little, proceso de cambio en ocho pasos, enfoque a resultados).

B. Obtengan rédito de la ingenuidad y creatividad de cada participante (tormenta de ideas, brainwriting, seis sombreros, matrices).

Con esto, los equipos pueden aplicar el PE aplicando cinco enfoques:

A. Diferenciándose de los competidores y siendo, lógicamente, mejor que ellos. PE significa encontrar un camino distinto para hacer lo que hacemos o adoptar un modelo de actividad distinto a partir de los existentes. 
Éxito del aprendizaje complejo a través del pensamiento estratégico

B. Emulando emprendedores. PE significa escanear las situaciones en busca de oportunidades mirando más allá de lo convencional. Intentar hacer algo mejor, más accesible, más barato, de manera diferente, más conveniente, más rápido, etc.

C. Encontrando nuevas oportunidades. PE significa moverse desde la perspectiva low-cost hacia el diseño de nuevos productos/modelos con herramientas como la visualización con Business model generation canvas.

D. Orientándose al futuro. PE significa explorar y diseñar mediante planificación de escenarios.

E. Siendo colaborativos. PE significa colaboración dentro del equipo y con el entorno y cualquier parte interesada (externalización, alianzas, cooperación).

\section{Resultados}

\subsection{Resultados de gestión}

En el curso 2014/2015 se obtuvieron los siguientes resultados a través de un ciclo de implantación, información, análisis, elaboración, difusión y evaluación:

A. El acuerdo con las Comisiones Académicas de cada Titulación (CATs) para impartir EPS en los 5 Títulos de Grado de la ETSID en el curso 2015/2016. La creación de materiales base adaptados a los diferentes grados.

B. 8 proyectos desarrollados por equipos creados a partir de 46 estudiantes de 10 países distintos que se detallan a continuación:

Don Tile: Design of a body tile

EASY ACCESS: The Automatic Door Lock System for Smart Homes Based on Face Recognition

EDUBOT: A Robot Designed for Educational Purposes

ELIT: Design of tiles for Scandinavia, Indonesia and Visual Impaired People

FUNATURE: Durable Furniture for Children

MUNDO: Innovative Proposal for Mestalla Atmosphere

NOMAD: Living in your car

SAMS: The Beat of Exploring

Los proyectos se coordinaron mediantes reuniones semanales y comunicaciones con diversas tecnologías, incluyendo la compartición de archivos (borradores, datos, etc.) en la nube virtual. Las sesiones comenzaron la primera semana de septiembre y las presentaciones finales se llevan a cabo pocos días antes de Navidad.

(c)) EY-NC-ND 2015, Universitat Politècnica de València

Congreso IN-RED (2015) 
C. Encuestas de satisfacción y de innovación abierta a los alumnos, profesores y observadores.

Se obtuvo un $100 \%$ de rendimiento académico en el EPS. La forma de trabajo en equipo contribuye a minimizar los riesgos de abandono, bajo rendimiento, etc.

Un 100\% de satisfacción de los estudiantes participantes en EPS. Los equipos están muy motivados por superar retos elegidos, que consideran próximos y relevantes.

Un 100\% de satisfacción de los profesores participantes en EPS. Todos consideran que su actuación como supervisores contribuye a su desarrollo profesional en el campo de la innovación educativa y del pensamiento estratégico.

Una lista de 12 profesores dispuestos a participar en futuros cursos y contribuciones críticas de 32 profesores.

El compromiso de tres nuevas universidades de impartir EPS en el curso 2015/2016: Glasgow Caledonian University, Hochschule Osnabrück y Nottingham Trent University.

El compromiso de 12 empresas de contribuir en el desarrollo de del EPS Valencia 105/2016.

\subsection{Resultados educativos}

En las siguientes tablas se muestran las técnicas educativas utilizadas, con indicación de un ejemplo de uso, y los resultados medios de los equipos y los individuos en los aspectos que se han valorado para observar la contribución a las competencias transversales.

Tabla 1. Técnicas utilizadas (divididas en cuatro fases) para el desarrollo del PE mientras se elabora el conjunto de proyectos multidisciplinares. Como ejemplo, las consideradas como cruciales por el equipo NOMAD están subrayadas.

\begin{tabular}{|c|c|c|c|}
\hline INMERSIÓN & ANÁLISIS & IDEACIÓN & PROTOTIPO \\
\hline Reencuadre/planteamiento & Diagrama de afinidad & Tormenta de ideas & P. en papel \\
\hline Investigación exploratoria & Mapa conceptual & Talleres de diseño & $\underline{\mathrm{P} \text { volumétrico }}$ \\
\hline Investigación de escritorio & Criterios Guía & Menú de ideas & Storyboard \\
\hline Entrevistas & Personas & Matriz de decisión & Escenarios \\
\hline Investigación Cultural & Mapa de empatía & & \\
\hline$\underline{\text { Sesiones generativas }}$ & $\underline{\text { Jornada de usuario }}$ & & \\
\hline \multicolumn{4}{|l|}{ Un día cualquiera } \\
\hline Sombra & & & \\
\hline
\end{tabular}

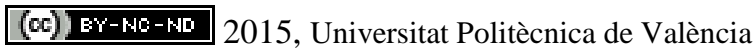

Congreso In-Red (2015) 
Éxito del aprendizaje complejo a través del pensamiento estratégico

Tabla 2. Técnicas empleadas, específicas del PE, para contribuir al proceso de design thinking seguido para elaborar los proyectos. Como ejemplo, se ha subrayado las consideradas como más interesantes por el equipo NOMAD.

\begin{tabular}{|c|c|c|c|}
\hline DÓNDE & A DÓNDE & QUÉ & CÓMO \\
\hline ESTAMOS & VAMOS & NECESITAMOS & LO HACEMOS \\
\hline Análisis DAFO & $\begin{array}{l}\text { Pensando en tres } \\
\text { horizontes }\end{array}$ & $\begin{array}{l}\text { Modelo de elementos } \\
\underline{\text { básicos }}\end{array}$ & $\begin{array}{l}\text { WBS y matriz de } \\
\text { responsabilidad }\end{array}$ \\
\hline Análisis DESTEP & Futuro perfecto & $\underline{\text { Planificación }}$ & Téc. presupuestarias \\
\hline $\begin{array}{l}\text { Business Model } \\
\text { Canvas }\end{array}$ & $\frac{\text { Business Model }}{\underline{\text { Canvas }}}$ & $\begin{array}{l}\text { Técnica de } \\
\text { Gordon/Little }\end{array}$ & $\begin{array}{l}\text { Preparación para el } \\
\text { cambio }\end{array}$ \\
\hline Mapa estratégico & Matriz CAME & Matriz de Ansoff & Enfoque a resultados \\
\hline Preguntas brutales & Estilos de decisión & Lista de atributos & Estilos comunicativos \\
\hline $\begin{array}{l}\text { Eligiendo el negocio } \\
\text { correcto }\end{array}$ & $\begin{array}{l}\text { Seis sombreros para } \\
\text { pensar }\end{array}$ & $\underline{\text { Análisis de inversión }}$ & $\begin{array}{l}\text { Proceso de cambio en } \\
\text { ocho pasos }\end{array}$ \\
\hline
\end{tabular}

Tabla 3. Resultados medios de la evaluación de los equipos $(n=8)$ por parte de los diferentes actores (3 supervisores, 5 externos, 37-42 pares). Máximo diez puntos.

\begin{tabular}{lccc}
\hline \multicolumn{1}{c}{ Competencia transversal } & Supervisores & Externos & Pares \\
\hline Recogen adecuadamente la información disponible & 9 & 8,5 & 7,5 \\
Aplican los conocimientos a la práctica de forma eficaz & 9,1 & 9,6 & 7,8 \\
Establecen un proceso para alcanzar los objetivos de forma & 8,7 & 9 & 8 \\
eficiente & & & \\
Utilizan correctamente técnicas de creatividad & 9 & 8,8 & 7,3 \\
Integran conocimiento complejo para generar ideas novedosas & 10 & 8,5 & 7,9 \\
Tienen en cuenta cómo afecta la innovación & 8,9 & 8,5 & 7,2 \\
Muestran una actitud emprendedora & 8 & 7,8 & 6,5 \\
Crean un prototipo ral completamente funcional & 8 & 9 & 7,9 \\
Comunican efizcamente la información técnica & 9 & 9,4 & 7,8 \\
\hline
\end{tabular}


Tabla 4. Resultados medios de la evaluación de los participantes $(n=46)$ por parte de los diferentes actores (2-3 supervisores, 3-6 externos, 45 pares). Máximo diez puntos.

\begin{tabular}{lccc}
\hline \multicolumn{1}{c}{ Competencia estratégica } & Supervisores & Externos & Pares \\
\hline Reencuadra/reformula adecuadamente & 9 & 8,6 & 7,5 \\
Abstrae lo importante & 9 & 9,3 & 7,2 \\
Escanea adecuadamente la información y las oportunidades & 8 & 8,9 & 9 \\
Muestra un pensamiento multivariante & 8 & 8,8 & 7,5 \\
Visualiza el futuro con facilidad & 8,2 & 8,4 & 7,5 \\
Usa la inducción eficicientemente & 8,5 & 8,5 & 8,9 \\
Valora adecuadamente las situaciones & 9 & 9,1 & 8,8 \\
\hline
\end{tabular}

\section{Conclusiones}

La experiencia de 10 años impartiendo EPS ha resultado en el liderazgo de una red internacional activa y en continuo crecimiento debido al interés en intercambiar profesores, estudiantes y experiencias adaptadas a las necesidades del siglo XXI. El EPS constituye un atractivo singular para los estudiantes de intercambio lo que permite fortalecer las relaciones bilaterales con prestigiosas universidades.

La participación de los profesores en EPS contribuye a su desarrollo en el campo de la innovación educativa. La multiculturalidad, la multidisciplinariedad, la comunicación y el trabajo en equipo se suman al aspecto estratégico. EPS está en continua evolución y mejora gracias, fundamentalmente, al proceso de innovación abierta.

El análisis de la literatura científica y de las opiniones de los agentes de interés en educación superior muestra que el pensamiento estratégico se puede aprender en edad universitaria, es muy recomendable para alcanzar la competencia experta y resulta fundamental para la empleabilidad.

El conjunto de técnicas de creatividad y estrategia puesto en práctica con los equipos EPS contribuyen al desarrollo de muchas competencias transversales, que se sintetizan en gran medida en el desarrollo del pensamiento estratégico y en los resultados obtenidos, tanto proyectuales como en las actividades propuestas. El escenario creado (técnicas, actividades, grupo internacional/multicultural, metacognición, evaluación 360) favorece los logros expuestos. 
Éxito del aprendizaje complejo a través del pensamiento estratégico

Este trabajo describe la incorporación del pensamiento estratégico a la educación superior con el fin de contribuir al aprendizaje complejo. El pensamiento estratégico es una habilidad valiosa en el actual escenario mundial, caracterizado por su globalidad e incertidumbre. La sociedad demanda graduados con capacidad de innovación continua y pensamiento estratégico con el fin de crear y mantener ventajas respecto a la competencia y progreso continuo. El pensamiento estratégico en la organización alienta a considerar ideas no convencionales y formular ideas en torno a un objetivo común y compartido.

Los autores agradecen a la UPV la concesión del Proyecto de Innovación y Mejora Educativa B33/14 y a la familia EPS su colaboración en la últimas dos décadas.

\section{Referencias}

AENOR (2006). Gestión de la I+D+i. Requisitos del sistema de gestión de la $I+D+i$. UNE 166002: 2006 EX. Madrid: AENOR.

BONN, I. (2001). "Developing strategic thinking as a core competency" en Management Decision, 39(1), 63-70.

CASTEJÓN, J. L., GILAR, R. y PÉREZ, A. M. (2006). “Aprendizaje complejo: el papel del conocimiento, la inteligencia, motivación y estrategias de aprendizaje” en Psicothema, Vol 18 (4), 679-685.

CHRISTENSEN, C. (1997). "Making Strategy: Learning by Doing” en Harvard Business Review 75(6), 141-156.

EDWARDS-SCHACHTER, M. et al. (2015). "Disentangling competences: Interrelationships on creativity, innovation and entrepreneurship" en Thinking Skills \& Creativity. Volume 16, June 2015, Pages 27-39.

GOLDMAN, E. et al. (2009). "Experiences to Develop the Ability to Think Strategically" en Journal of Healthcare Management, 54(6), 403-416.

GRANT, R.M. (2013). Contemporary Strategy Analysis. Edinburgh: Wiley.

HAMEL, G., \& PRAHALAD, C.K. (2005). "Strategic Intent” en Harvard Business Review, July-August 2005, 148-161.

LIEDTKA, J. (1998). Strategic thinking: can it be taught? Long Range Planning, 31(3), 481-487.

LINKOW, P. (1999). "What Gifted Strategic Thinkers Do” en Training \& Development, $53,7,34-37$.

LLOVET, J. (1979). Ideologia y metodología del diseño. Barcelona: Gustavo Gili.

OLTRA, M.J. et al. (2014). Autoevaluación de la innovación. Versión adaptada a la universidad. Madrid: Club Excelencia en Gestión.

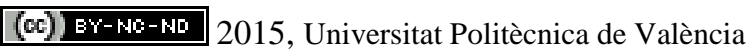

Congreso IN-RED (2015) 
SHAFIRI, E. (2012). "Strategic Thinking; a practical view” en Ideal type of Management, 1(1), 71-84.

SLOAN, J. (2006). Learning to Think Strategically. Oxford, UK.

SWEET, S. y MEIKSINS, P. (2013). Changing Contours of Work: Job and Opportunities in the New Economy. New Delhi: Sage Publications.

VEST, Ch (2012). Educating Engineers for 2020 and Beyond. Washington: National Academy of Engineering.

WARD, P., ERICSSON, K. A., y WILLIAMS, A. M. (2013). "Complex perceptualcognitive expertise in a simulated task environment" en Journal of Cognitive Engineering and Decision Making, 7, 231-254.

WELLS, S. (2001). “To Plan, Perchance, to Think; Aye, There’s the Rub” En Information Outlook, 5, $9: 8-12$.

ZAND, D.E. (2010). "Drucker's strategic thinking process: three key techniques” en Strategy\&leadership, 38(3), 23-28. 